\title{
Is There a Preferential Interaction between Cholesterol and Tryptophan Residues in Membrane Proteins? ${ }^{\dagger}$
}

\author{
Andrea Holt, ${ }^{*}$, Rodrigo F. M. de Almeida, ${ }^{\S}$ Thomas K. M. Nyholm, ${ }^{\ddagger}$ Luís M. S. Loura, ${ }^{\perp}$ Anna E. Daily, ${ }^{@}$ \\ Rutger W. H. M. Staffhorst, ${ }^{\circ}$ Dirk T. S. Rijkers, ${ }^{+}$Roger E. Koeppe II, ${ }^{@}$ Manuel Prieto, ${ }^{\S}$ and J. Antoinette Killian ${ }^{\ddagger}$ \\ Chemical Biology and Organic Chemistry, Bijvoet Center for Biomolecular Research, Utrecht University, Padualaan 8, \\ 3584 CH Utrecht, The Netherlands, Medicinal Chemistry and Chemical Biology, Utrecht Institute for Pharmaceutical Sciences, \\ Utrecht University, Sorbonnelaan 16, 3584 CA Utrecht, The Netherlands, Centro de Química-Física Molecular, \\ Complexo Interdisciplinar, Instituto Superior Técnico, Av. Rovisco Pais, 1049-001 Lisbon, Portugal, Faculdade de \\ Farmácia da Universidade de Coimbra, 3000-295 Coimbra, Portugal, and Department of Chemistry and Biochemistry, \\ University of Arkansas, Fayetteville, Arkansas 72701
}

Received November 9, 2007; Revised Manuscript Received December 12, 2007

\begin{abstract}
Recently, several indications have been found that suggest a preferential interaction between cholesterol and tryptophan residues located near the membrane-water interface. The aim of this study was to investigate by direct methods how tryptophan and cholesterol interact with each other and what the possible consequences are for membrane organization. For this purpose, we used cholesterol-containing model membranes of dimyristoylphosphatidylcholine (DMPC) in which a transmembrane model peptide with flanking tryptophans [acetyl-GWW(LA) 8 LWWA-amide], called WALP23, was incorporated to mimic interfacial tryptophans of membrane proteins. These model systems were studied with two complementary methods. (1) Steady-state and time-resolved Förster resonance energy transfer (FRET) experiments employing the fluorescent cholesterol analogue dehydroergosterol (DHE) in combination with a competition experiment with cholesterol were used to obtain information about the distribution of cholesterol in the bilayer in the presence of WALP23. The results were consistent with a random distribution of cholesterol which indicates that cholesterol and interfacial tryptophans are not preferentially located next to each other in these bilayer systems. (2) Solid-state ${ }^{2} \mathrm{H}$ NMR experiments employing either deuterated cholesterol or indole ring-deuterated WALP23 peptides were performed to study the orientation and dynamics of both molecules. The results showed that the quadrupolar splittings of labeled cholesterol were not affected by an interaction with tryptophan-flanked peptides and, vice versa, that the quadrupolar splittings of labeled indole rings in WALP23 are not significantly influenced by addition of cholesterol to the bilayer. Therefore, both NMR and fluorescence spectroscopy results independently show that, at least in the model systems studied here, there is no evidence for a preferential interaction between cholesterol and tryptophans located at the bilayer interface.
\end{abstract}

Biological membranes are composed of a variety of different lipids and contain a huge diversity of membrane proteins. They are very heterogeneous systems and often include membrane microdomains that can have compositions different from that of the bulk membrane and that are important for some membrane proteins to fulfill their func-

\footnotetext{
$\dagger$ This research project has been supported by a Marie Curie Early Stage Research Training Fellowship from the European Community's Sixth Framework Program for A.H. under Contract Biomem-MESTCT 2004-007931, by a grant from the Sigrid Juselius Foundation to T.K.M.N., by NIH Grant RR15569 from the United States Public Health Service to R.E.K., and by postdoctoral fellowships for R.F.M.d.A. from Fundação para a Ciência e a Tecnologia, Portugal, under Programs POCTI (SFRH/BPD/178842/2004) and PIDDAC (SFRH/BPD/32210/ 2006).

* To whom correspondence should be addressed. E-mail: a.holt@uu.nl. Phone: +31-30-2533342. Fax: +31-30-2533969.

¥ Bijvoet Center for Biomolecular Research, Utrecht University.

$\S$ Instituto Superior Técnico.

" Present address: Department of Biochemistry and Pharmacy, Åbo Akademi University, Artillerigatan 6A, 20520 Turku, Finland.

${ }^{\perp}$ Faculdade de Farmácia da Universidade de Coimbra.

@ University of Arkansas.

+ Utrecht Institute for Pharmaceutical Sciences, Utrecht University.
}

tions (1). Often the formation of these membrane microdomains involves local enrichment of cholesterol $(2,3)$.

Cholesterol can be found in membranes of eukaryotic organisms and is a major constituent in some types of membranes (4). It is thought to have a dual function in the membrane (5). On one hand, it has an ordering effect on acyl chains of surrounding (phospho)lipids which results in a more condensed bilayer. Membrane domains with an increased cholesterol content, also referred to as lipid rafts, therefore have an increased bilayer thickness. On the other hand, cholesterol introduces negative membrane curvature and is able to fill voids in the bilayer due to its bulky body. The presence of cholesterol thus affects several membrane properties which can also influence the function and distribution of membrane proteins.

Membrane proteins perform many essential functions in the cell such as transport, signaling, catalysis, and transduction of energy. The transmembrane domains of these proteins are hydrophobic and frequently include aromatic residues such as tryptophan and tyrosine located at the lipid-water interface $(6,7)$. In particular, these interfacial tryptophans 
Table 1: Amino Acid Sequences of the Model Peptides

\begin{tabular}{cl}
\hline peptide & \multicolumn{1}{c}{ amino acid sequence } \\
\hline WALP23 & acetyl-GWWLALALALALALALALALWWA-amide \\
KALP23 & acetyl-GKKLALALALALALALALALKKA-amide \\
WLP23 & acetyl-GWWLLLLLLLLLLLLLLLLLWWA-amide \\
KLP23 & acetyl-GKKLLLLLLLLLLLLLLLLLKKA-amide \\
WALP23- $d_{5}$-Trp2 & acetyl-GWWLALALALALALALALALWWA-ethanolamine ${ }^{a}$ \\
WALP23- $d_{5}$-Trp3 & acetyl-GWWLALALALALALALALALWWA-ethanolamine $^{a}$ \\
\hline${ }^{a}$ Bold letters indicate positions labeled with indole-deuterated $d_{5}$-Trp.
\end{tabular}

${ }^{a}$ Bold letters indicate positions labeled with indole-deuterated $d_{5}$-Trp.

are believed to anchor transmembrane domains of membrane proteins in the bilayer and to help them to adopt correct positioning needed for proper functioning $(8,9)$. Tryptophans at the membrane interface are also thought to have an important role in folding and assembly of transmembrane complexes of membrane proteins (7).

The indole ring makes tryptophan the bulkiest amino acid among all 20 amino acids naturally occurring in proteins and allows amphipathic interactions, including imino group hydrogen bonding, specific dipolar interactions, cation $-\pi$ interactions, and hydrophobic interactions $(7,10,11)$. The special character of the indole ring provides the potential to interact with a variety of molecules. The rigid indole ring of tryptophan is often found stacking with other rigid ring structures than found in nucleic acids or other amino acids, including phenylalanine and tyrosine.

Cholesterol also has a complex rigid ring structure which could favorably interact with the indole ring of tryptophans. If such an interaction occurs and if it is sufficiently favorable, it could lead to a preferential interaction between cholesterol and tryptophans located at the bilayer interface. Indeed, several indications have been found for such an interaction. In a study by Gasset et al. (12), the authors proposed the formation of a complex between cholesterol and the tryptophan-rich gramicidin A driven by hydrophobic interactions. Carozzi et al. (13) found that a cysteine to tryptophan mutation in the membrane protein caveolin-3 affects the distribution of cholesterol in the membrane. This naturally occurring $\mathrm{C} 71 \mathrm{~W}$ mutation was observed to increase the affinity of caveolin-3 for cholesterol and thereby prevent the release of cholesterol from caveolae to noncaveolar membrane domains. In another study, Santiago et al. (14) found that a cysteine to tryptophan mutation alters the function of the nicotinic acetylcholine receptor AChR. This $\alpha \mathrm{C} 418 \mathrm{~W}$ mutation which is located in a lipid-exposed transmembrane segment dramatically enhanced the response of the receptor to cholesterol modulation. Finally, Van Duyl et al. (5) observed a synergistic effect between cholesterol and tryptophan-flanked transmembrane peptides in lipid phase behavior. They found that model membranes composed of unsaturated phospholipids and cholesterol form an isotropic phase upon incorporation of tryptophan-flanked peptides. This was not observed for model peptides in which the flanking tryptophans had been replaced with lysines or histidines, and these results led the authors to propose a preferential interaction between cholesterol and interfacial tryptophans.

In spite of these indications for a preferential interaction between cholesterol and tryptophans, there is little known about the interaction and the possible consequences for membrane organization. Here, we investigated this issue by employing tryptophan-flanked model peptides which were incorporated into bilayers of phospholipids containing varying amounts of cholesterol. These peptides, called WALP peptides, consist of a stretch of alternating leucines and alanines flanked on both sides by a pair of tryptophans. These transmembrane peptides have been designed as well-defined models to gain insight into basic principles of membrane organization and have been studied extensively throughout the past decade $(9,15-17)$. They have often been used to mimic transmembrane segments of membrane proteins (16) and readily integrate into bilayers under formation of stable transmembrane $\alpha$-helices.

To study the interaction between cholesterol and the interfacial tryptophans of WALP peptides, we used a 2-fold approach employing fluorescence spectroscopy and $\mathrm{NMR}^{1}$ spectroscopy. Fluorescence experiments using Förster resonance energy transfer (FRET) between tryptophan and the fluorescent cholesterol analogue dehydroergosterol (DHE) were used to obtain information about the distribution of cholesterol in the bilayer with respect to the peptides. Solidstate ${ }^{2} \mathrm{H}$ NMR measurements using either deuterated cholesterol or tryptophan-deuterated WALP peptides were performed to observe possible changes in the motion and orientation of cholesterol and tryptophan. The results indicate that cholesterol remains homogeneously distributed in the bilayer in the presence of tryptophan-flanked peptides and that the dynamics and orientation of neither cholesterol nor tryptophans located at the bilayer interface are influenced by the presence of the other molecule.

\section{MATERIALS AND METHODS}

Materials. Unlabeled WALP23, KALP23, WLP23, and KLP23 peptides (for amino acid sequences, see Table 1) were synthesized using Fmoc/tBu solid-phase synthesis as described elsewhere for related KALP peptides (18). Deuteriumlabeled L-tryptophan (indole- $d_{5}, 98 \%$ ) was purchased from Cambridge Isotopes Laboratories Inc. (Andover, MA), and Fmoc (9-fluorenylmethyloxycarbonyl) was used to protect its amino functionality as described by Ten Kortenaar et al. (19) before being used in the peptide synthesis. The deuterium-labeled WALP23 peptides were synthesized according to the procedure described in ref 20 to prevent exchange of deuterons located at the indole ring against hydrogens in the presence of trifluoroacetic acid (TFA) (21). Due to the use of ethanolamine as a cleavage agent, the C-terminus of the deuterium-labeled WALP23 peptide is capped by ethanolamine instead of an amide. Indole ring-

${ }^{1}$ Abbreviations: DHE, dehydroergosterol; DMPC, dimyristoylphosphatidylcholine; FRET, Förster resonance energy transfer; KALP23 and KLP23, lysine-flanked $\alpha$-helical model peptides; LUV, large unilamellar vesicle; NMR, nuclear magnetic resonance; TFE, trifluoroethanol; WALP23 and WLP23, tryptophan-flanked $\alpha$-helical peptides. 
deuterated tryptophans were introduced at position 2 or 3 of the WALP23 peptide. The identity of the synthesized peptides was verified by mass spectrometry, and the purity was analyzed by HPLC using a C4 reverse phase HPLC column with an aqueous phosphorous acid/triethylamine buffer at $\mathrm{pH} 2.25$, according to procedures described in ref 22 . The purity of peptides used in this study was generally better than $95 \%$.

Cholesterol and 1,2-dimyristoyl-sn-glycero-3-phosphocholine (DMPC) were purchased from Avanti Polar Lipids Inc. (Alabaster, $\mathrm{AL}$ ) and used without further purification. $\beta-\left(d_{6}-2,2,3,4,4,6\right)$ Cholesterol was purchased from Medical Isotopes Inc. (Pelham, NH). 2,2,2-Trifluoroethanol (TFE) was obtained from Merck (Darmstadt, Germany). Ergosta5,7,9(11),22-tetraen-3 $\beta$-ol (DHE, >96\%) was purchased from Sigma Aldrich. All lipids were used without further purification. All other chemicals used were analytical grade. Deuterium-depleted water for NMR samples was obtained from Cambridge Isotope Laboratories, Inc. All other water used during sample preparation was deionized and purified with a Milli-Q Gradient Water purification system from Millipore Corp. (Billerica, MA).

Methods. (i) Fluorescence Spectroscopy. Stock solutions of ca. $20 \mathrm{mM}$ DMPC in chloroform were prepared, and the lipid concentration was determined by a phosphorus assay (23). Stock solutions of WALP23 and DHE were prepared with a concentration of ca. $200 \mu \mathrm{M}$ in TFE and $\mathrm{MeOH}$, respectively. The concentration of WALP23 and DHE was determined by absorption spectroscopy using extinction coefficients of $22400 \mathrm{M}^{-1} \mathrm{~cm}^{-1}$ at $280 \mathrm{~nm}$ for WALP23 (24) and $10500 \mathrm{M}^{-1} \mathrm{~cm}^{-1}$ at $324 \mathrm{~nm}$ for DHE (25). Cholesterol stock solutions were prepared by weight with a concentration of $5 \mathrm{mM}$ in chloroform.

All fluorescence experiments were performed with a constant peptide/phospholipid molar ratio of 1/200 and a final peptide concentration of $5 \mu \mathrm{M}$. First, appropriate amounts of peptide and phospholipid were mixed in solution and distributed over a number of tubes. Then varying amounts of DHE (0-8 mol \%) were added to the tubes. For competition experiments, additionally equimolar amounts of cholesterol were added. After thorough mixing, the organic solvents were evaporated under a nitrogen flow and were further removed under vacuum overnight (ca. $1 \times 10^{-2}$ mbar). Then the samples were hydrated with buffer [ $25 \mathrm{mM}$ HEPES and $100 \mathrm{mM} \mathrm{NaCl}(\mathrm{pH} 7.0)]$ and incubated for $\sim 30$ min. Large unilamellar vesicles (LUVs) were produced by first freeze-thawing the samples at least 10 times and subsequent extrusion through inorganic membrane filters with a pore size of $200 \mathrm{~nm}$ (Anotop 10; Whatman International Ltd., Maidstone, England).

For steady-state fluorescence measurements, the samples $(1.2 \mathrm{~mL})$ were directly after preparation transferred to a 10 mm quartz cuvette. All measurements were performed using a SLM-Aminco SPF-500 C fluorimeter (Jobin Yvon, Edison, $\mathrm{NJ}$ ) and were carried out at $30^{\circ} \mathrm{C}$ to ensure that the lipid bilayers are in the fluid phase. The samples were allowed to equilibrate in the fluorimeter for at least $5 \mathrm{~min}$ under continuous stirring before the measurements. The tryptophans of WALP23 were excited at $280 \mathrm{~nm}$, and emission from tryptophan and DHE was recorded from 300 to $525 \mathrm{~nm}$ using a bandwidth of $5 \mathrm{~nm}$ for both the excitation and emission channel. All emission spectra were corrected for instrument specific deviations using a standard emission spectrum of L-tryptophan (26). Samples without peptide and DHE were used to correct for light scattering of the vesicle suspensions.

Fluorescence decay measurements were carried out on a time-correlated single-photon timing system which is described elsewhere $(27,28)$. All time-resolved fluorescence measurements were performed at $30{ }^{\circ} \mathrm{C}$. For the FRET experiments, the donor (Trp in WALP23) was excited at 290 $\mathrm{nm}$ to minimize absorption of the acceptor (DHE). Tryptophan emission was collected at $330 \mathrm{~nm}$ using the magic angle relative to the vertically polarized excitation beam, and to avoid scattered excitation, a cutoff filter was added to the monochromator. The collection bandwidth was set to $20 \mathrm{~nm}$ to prevent acceptor emission from reaching the detector. The fluorescence decays were obtained with an accumulation of 20000 counts on the peak channel and time scales ranging from $22 \mathrm{ps} / \mathrm{channel}$ for donor only to $12 \mathrm{ps} / \mathrm{channel}$ for high acceptor concentrations.

(ii) Analysis of Fluorescence Data. Data analysis was carried out using a nonlinear, least-squares iterative convolution method based on the algorithm of Marquardt (29). The decay curves obtained for different acceptor concentrations were analyzed using a global analysis approach with linkage of lifetimes and ratios of preexponential factors (27). The quality of the fit was judged from $\chi^{2}$ values of the individual experiments, the global $\chi^{2}$ value $\left(\chi_{\mathrm{G}}{ }^{2}\right)$, and weighted residuals and autocorrelation plots.

The critical distance for FRET, the Förster distance $\left(R_{0}\right)$, was calculated by

$$
R_{0}=0.2108\left[\kappa^{2} n^{-4} \Phi_{\mathrm{D}} \int_{0}^{\infty} \lambda^{4} I(\lambda) \epsilon(\lambda) \mathrm{d} \lambda\right]^{1 / 6}
$$

where $\kappa$ is the orientation factor for dipole-dipole coupling of the donor and acceptor, $\Phi_{\mathrm{D}}$ is the quantum yield of the donor in the absence of the acceptor, $n$ is the refractive index of the medium, $\lambda$ is the wavelength expressed in nanometers, $I(\lambda)$ is the normalized emission spectrum of the donor, and $\epsilon(\lambda)$ is the molar absorption spectrum of the acceptor in $\mathbf{M}^{-1}$ $\mathrm{cm}^{-1}(30)$. The resulting Förster distance has units of angstroms. An $n=1.4$ refractive index was used for the bilayer interface $(30,31)$ which is an average value between $n=1.5$ for the bilayer interior (32) and $n=1.33$ for an aqueous environment. For the orientation factor $\kappa^{2}$, a value of $2 / 3$ was used which represents the dynamic isotropic limit; i.e., the donor and acceptor undergo fast reorientation during the lifetime of the excited state of the donor $(31,33)$. This value is commonly used for FRET experiments performed in bilayers $(27,34)$.

For FRET within one leaflet of the bilayer, the decay of donor fluorescence in the presence of an acceptor for unlinked and randomly distributed donor and acceptor was derived in ref 35

$$
\begin{aligned}
\rho_{\text {cis }}(t)=\exp \left(-\pi R_{0}{ }^{2} n \gamma\left[\frac{2}{3},\left(\frac{R_{0}}{R_{\mathrm{e}}}\right)^{6}\left(\frac{t}{\langle\tau\rangle}\right)\right]\left(\frac{t}{\langle\tau\rangle}\right)^{1 / 3}+\right. \\
\left.\pi R_{\mathrm{e}}{ }^{2} n\left\{1-\exp \left[-\left(\frac{R_{0}}{R_{\mathrm{e}}}\right)^{6}\left(\frac{t}{\langle\tau\rangle}\right)\right]\right\}\right)
\end{aligned}
$$

where $n$ is the surface density of acceptors, $R_{\mathrm{e}}$ is the radius of exclusion of acceptors around the donor, and the average lifetime $\langle\tau\rangle=\sum_{i} \alpha_{i} \tau_{i}^{2} / \sum_{i} \alpha_{i} \tau_{i}$. For the calculations of the FRET 
efficiency between the tryptophans of WALP23 and DHE in DMPC bilayers, the radius of exclusion $\left(R_{\mathrm{e}}\right)$ was estimated to be $7.5 \AA$, being the sum of the radii of tryptophan and DHE calculated from the square root over the area of the molecules in the bilayer. The incomplete Gamma function $\gamma$ is defined as $\gamma[x, y]=\int_{0}^{y} z^{x-1} \exp (-z) \mathrm{d} z$.

For FRET to the opposing bilayer leaflet, the decay of donor fluorescence was derived in ref 31

$$
\begin{aligned}
\rho_{\text {trans }}(t)=\exp \left(-2 \pi w^{2} n \int_{0}^{w /\left(w^{2}+R_{\mathrm{e}}^{2}\right)^{1 / 2}} \times\right. \\
\left.\quad\left\{1-\exp \left[-\left(\frac{R_{0}}{R_{\mathrm{e}}}\right)^{6}\left(\frac{t}{\langle\tau\rangle}\right) \alpha^{6}\right]\right\} \alpha^{-3} \mathrm{~d} \alpha\right)
\end{aligned}
$$

where $w$ is the interplanar distance between the donor and acceptor which was considered to be $23 \AA$ on the basis of molecular models.

The decay of donor fluorescence in the presence of acceptor molecules in both leaflets is then described by

$$
i_{\mathrm{DA}}(t)=i_{\mathrm{D}}(t) \rho_{\text {cis }}(t) \rho_{\text {trans }}(t)
$$

Finally, the FRET efficiency is calculated by numerical integration of

$$
E=1-\frac{\int_{0}^{\infty} i_{\mathrm{D}}(t) \rho_{\text {cis }}(t) \rho_{\text {trans }}(t) \mathrm{d} t}{\langle\tau\rangle_{\mathrm{D}}}
$$

where $i_{\mathrm{D}}(t)=\sum_{i} \alpha_{i} \exp \left[-\left(t / \tau_{\mathrm{D} i}\right)\right]$ describes the fluorescence decay of the donor in the absence of acceptor and $\langle\tau\rangle_{\mathrm{D}}=$ $\sum_{i} \alpha_{i} \tau_{i}$ corresponds to the donor lifetime weighted by quantum yield.

(iii) NMR Spectroscopy. Phospholipid and (deuterated) cholesterol stock solutions were prepared as described above. For NMR experiments employing deuterated cholesterol, typically $2 \mu \mathrm{mol}$ of deuterated cholesterol and $100 \mu \mathrm{mol}$ of phospholipids were used per sample. The lipids were mixed in solution, and organic solvents were removed as described above. Deuterium-depleted buffer was prepared by lyophilization of a small amount of buffer [25 mM HEPES and $100 \mathrm{mM} \mathrm{NaCl}$ (pH 7.0)] prepared with Milli-Q water which was rehydrated with the same amount of deuterium-depleted water. The samples were first hydrated with Milli-Q water $(1 \mathrm{~mL})$ and lyophilized. Then the powder was transferred to the smaller NMR tubes and hydrated using $100 \mu \mathrm{L}$ of deuterium-depleted buffer. The NMR tubes were sealed with a silicon stopper under a $\mathrm{N}_{2}$ atmosphere and freeze-thawed 10 times to promote sample homogeneity. Samples with a higher cholesterol concentration in the bilayer were supplemented with undeuterated cholesterol. For peptide-containing samples, $1 \mu \mathrm{mol}$ of the indicated peptide was weighed, dissolved in TFE $(0.5 \mathrm{~mL})$, and added to the lipid mixture.

The NMR experiments employing tryptophan-deuterated WALP peptides were performed on oriented samples. Stock solutions of deuterated WALP peptides were prepared with a concentration of ca. $500 \mu \mathrm{M}$ in TFE. For each oriented sample, typically $2 \mu \mathrm{mol}$ of deuterated peptide and $50 \mu \mathrm{mol}$ of phospholipids were mixed in solution. For samples also containing cholesterol, $2 \mu \mathrm{mol}$ of the sterol was added to the mixture. Organic solvents were removed as described above, and the sample was redissolved in a mixture of

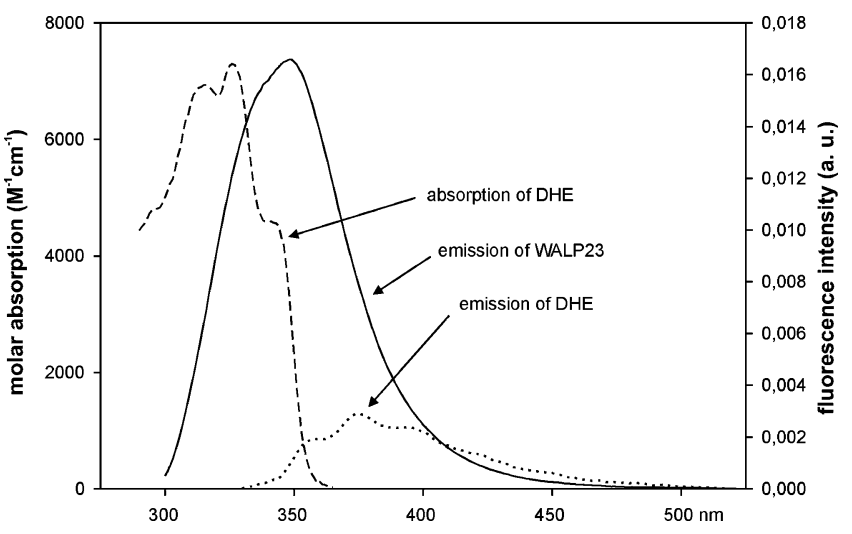

FIGURE 1: Spectral overlap for the FRET pair with WALP23 as the donor and DHE as the acceptor in LUVs of DMPC at pH 7.0: (- - ) absorption spectrum of DHE with a molar absorption coefficient $\epsilon$ of $7300 \mathrm{M}^{-1} \mathrm{~cm}^{-1}$ at maximal absorption at $324 \mathrm{~nm}$, $(-)$ fluorescence emission spectrum of WALP23, and $(\cdots)$ fluorescence emission spectrum of DHE. The spectra were recorded at a temperature of $30{ }^{\circ} \mathrm{C}$. The excitation wavelength was $280 \mathrm{~nm}$ for WALP23 and $324 \mathrm{~nm}$ for DHE.

methanol and chloroform (1/1, v/v, $2 \mathrm{~mL})$. This mixture was distributed over 50 glass platelets $(4.8 \mathrm{~mm} \times 23 \mathrm{~mm} \times$ 0.06-0.08 mm; Marienfeld Laboratory Glassware, LaudaKönigshafen, Germany), and solvents were evaporated as described. Then the platelets were stacked into a square glass cuvette and if necessary supplemented with empty glass platelets to ensure a tight fit. The sample was hydrated with deuterium-depleted water to $40 \%$ (w/w) hydration, and the cuvette was sealed immediately under a nitrogen atmosphere using a glass plate and quick-drying epoxy glue. To promote alignment of the bilayers along the glass plates, the samples were incubated at $37^{\circ} \mathrm{C}$ for at least 3 days before NMR measurements.

NMR experiments were carried out on a Bruker Avance $500 \mathrm{MHz}$ wide bore spectrometer (Bruker Biospin, Karlsruhe, Germany). All NMR measurements were performed at $40{ }^{\circ} \mathrm{C}$. The samples were allowed to equilibrate for at least 10 min before measurements. The bilayer alignment of the oriented samples was checked by ${ }^{31} \mathrm{P}$ NMR experiments as described in ref $36 .{ }^{2} \mathrm{H}$ NMR experiments were performed at $76.78 \mathrm{MHz}$ using a quadrupolar echo sequence (37), an echo delay of $50 \mu \mathrm{s}$, and a relaxation time of $100 \mathrm{~ms}$. For ${ }^{2} \mathrm{H}$ NMR measurements on the samples containing deuterated cholesterol, 400000 scans were acquired. For the oriented samples containing deuterated peptides, $2.4 \times 10^{6}$ and 1.6 $\times 10^{6}$ scans were acquired for the $0^{\circ}$ and $90^{\circ}$ orientations of the bilayer normal to the magnetic field, respectively.

\section{RESULTS}

FRET Experiments. FRET measurements were carried out to obtain information about the lateral distribution of sterols in the bilayer in the presence of tryptophan-flanked peptides. Since cholesterol itself is not fluorescent, we used the fluorescent cholesterol analogue DHE. The fluorescence characteristics of WALP23 and DHE in bilayers of DMPC are shown in Figure 1. The emission of the tryptophans (solid line) overlaps with the absorbance of DHE (dashed line) in the range of 300-355 $\mathrm{nm}$ which allows FRET between these molecules, whereby the tryptophans of WALP23 serve as 

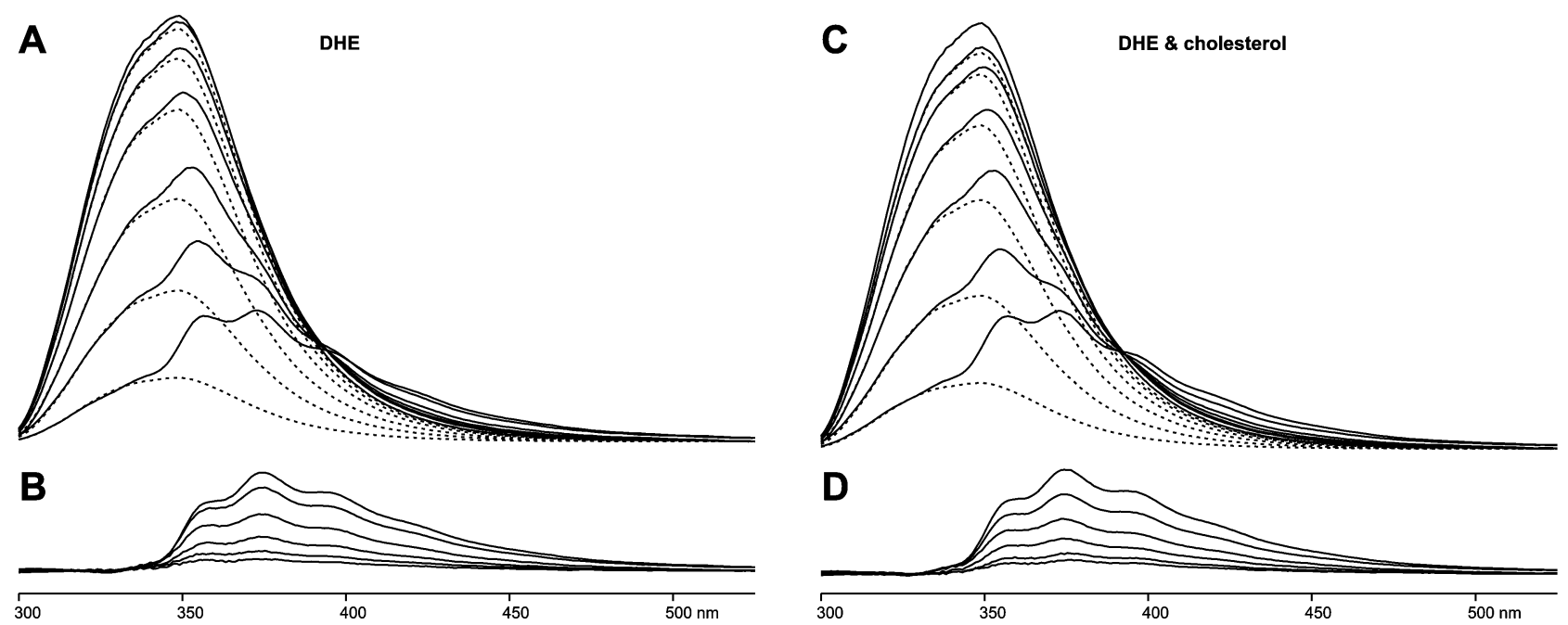

FIGURE 2: Fluorescence emission spectra of WALP23 and of DHE in bilayers of DMPC containing different concentrations of sterols at $\mathrm{pH} 7.0$ and $30^{\circ} \mathrm{C}$. (A) The solid lines are spectra from FRET experiments using increasing DHE concentrations (from top to bottom, 0, $0.25,0.5,1,2,4$, and $8 \mathrm{~mol} \%$ ) added to the lipid fraction. The dashed lines show the fluorescence emission of the tryptophans of WALP23, obtained by decomposition. (B) Fluorescence emission of DHE (from top to bottom, 8, 4, 2, 1, 0.5, and 0.25 mol \%), obtained by decomposition of the spectra in panel A. (C) Samples as in panel A but with equimolar amounts of DHE and cholesterol added to the lipid fraction. (D) Fluorescence emission of DHE in the presence of equimolar amounts of cholesterol, obtained by decomposition of the spectra in panel C. In all samples, the excitation wavelength was $280 \mathrm{~nm}$ and the final concentration of WALP23 was $5 \mu \mathrm{M}$ using a peptide/lipid ratio of $1 / 200$.

the donor and DHE serves as the acceptor. For DHE, the maximal molar extinction coefficient in DMPC bilayers was determined to be $7300 \mathrm{M}^{-1} \mathrm{~cm}^{-1}$ at $324 \mathrm{~nm}$ by comparison with absorption of DHE in methanol using a molar extinction coefficient of $10500 \mathrm{M}^{-1} \mathrm{~cm}^{-1}$ at $324 \mathrm{~nm}$ (25). The overlap integral was solved on the basis of the absorption spectrum of DHE and the normalized emission spectrum of WALP23 as shown in Figure 1. The quantum yield of the tryptophans of WALP23 in DMPC bilayers was determined to be 0.106 using tryptophan in water as a standard (30) and an excitation wavelength of $280 \mathrm{~nm}$. On the basis of these parameters, the characteristic Förster distance for the FRET pair, WALP23 and DHE, was calculated to be $20 \AA$ according to eq 1.

Distribution of Cholesterol in the Membrane. Steady-state fluorescence spectra were recorded on DMPC bilayers containing WALP23 peptides and increasing concentrations of 0-8 mol \% DHE in the lipid fraction (Figure 2A). From the spectral line shapes, it is clear that as the surface density of DHE in the bilayer increases, the intensity of the emission due to the tryptophans of WALP23 (dashed lines in the top panel) decreases. A concomitant increase in the emission of the acceptor is observed (solid lines in the bottom panel), which is mainly the sensitized emission of DHE (due to FRET from WALP23) and a minor contribution of the direct excitation of DHE at $280 \mathrm{~nm}$. Because the emission spectra of WALP23 and DHE overlap to a great extent (see Figure 1 ), the recorded spectra need to be decomposed into the emission of the donor and the emission of the acceptor to calculate the FRET efficiency from the integrated area of the spectrum. In principle, the calculation could also be performed from the steady-state intensity at a given wavelength at which there is no emission from the acceptor. However, via decomposition of the spectra, it is possible to check whether the shape of the emission spectra remains the same, ruling out the presence of other photophysical processes. In addition, the method is more reliable since the area under the spectrum is directly proportional to the quantum yield of fluorescence even if small spectral shifts are taking place. Since the emission between 300 and 330 $\mathrm{nm}$ originates exclusively from the tryptophans of WALP23, we fitted the emission spectrum of WALP23 in the absence of acceptor DHE in the range between 300 and $330 \mathrm{~nm}$ to the emission spectra for different concentrations of DHE. The FRET efficiency was then calculated from the area integral ratio between the fitted spectrum and the original spectrum for donor only as $E=1-\phi_{\mathrm{DA}} / \phi_{\mathrm{D}}$, where $\phi$ is the quantum yield of the donor alone (D) or in the presence of acceptor (DA). Subtraction of the fitted spectrum from the total emission spectrum yielded the emission of DHE (Figure 2B).

The FRET efficiencies are plotted in Figure $3(O)$ as a function of the surface density of DHE in the bilayer which was calculated from the mole fraction of DHE in the bilayer using areas per lipid molecule of $59.5 \AA^{2}$ for DMPC (38) and $37.7 \AA^{2}$ for DHE and cholesterol (39). Condensation effects due to sterols present in the bilayer were assumed to be negligible at the low concentrations of sterols used here (39). The FRET efficiency in a bilayer, a quasi-twodimensional system, depends on the Förster distance of the FRET pair, the exclusion radius of the acceptor around the donor, and the amount and distribution of acceptor in the bilayer. The theoretical FRET efficiency for a random distribution of WALP23 and DHE in both leaflets of the bilayer was calculated on the basis of eqs 2 and 3 computed into eq 5. This curve is shown in Figure $3(-)$ for different theoretical values of the Förster distance. The experimentally determined FRET efficiencies for different surface densities of DHE match the theoretical FRET efficiency curve for the calculated Förster distance of $20 \AA$ ( $O$ in Figure 3$)$. If the acceptor were excluded from the vicinity of the donor, the experimental FRET efficiencies would be located below the theoretical FRET efficiency curve (see ref 40 for a nonhomogeneous distribution with acceptor excluded from donor). 


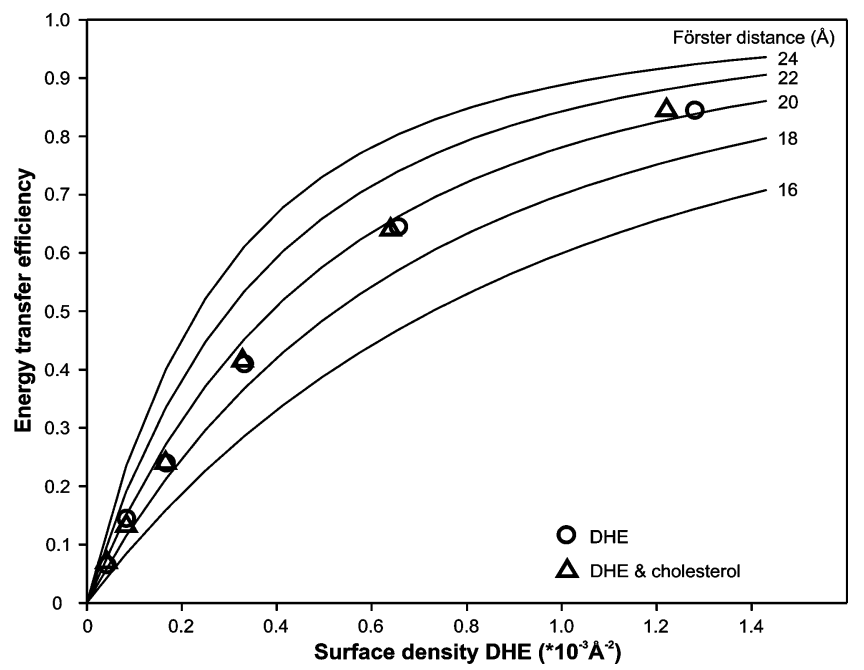

FIGURE 3: Theoretically calculated FRET effciency curves (eq 5) for different Förster distances based on the model for a random distribution of acceptor taking into account transfer two both leaflets (eqs 2 and 3) are shown as solid lines. FRET effciencies calculated from steady-state data (Figure 2) with WALP23 as the donor and DHE as the acceptor depending on the surface density of the acceptor in the bilayer in the presence of only DHE (O) and in the presence of the equimolar amounts of DHE and cholesterol $(\triangle)$.

Alternatively, if the acceptor is attracted to the donor, the experimental values would be located above the theoretical curve. Therefore, these results indicate that DHE is randomly distributed in the bilayer in the presence of tryptophanflanked peptides.

As a complementary approach, we also used time-resolved fluorescence techniques (41). Fluorescence decays were recorded for the same sample compositions used for the steady-state measurements described above (Figure $4 \mathrm{~A}$ ). With an increasing DHE concentration in the lipid bilayer, the intensity of tryptophan fluorescence is decaying faster due to an increasing level of FRET from the tryptophans to DHE. The fluorescence decay of WALP23 in DMPC at 30 ${ }^{\circ} \mathrm{C}$ shows multiexponential behavior and can be described by a sum of three exponentials with the following parameters: $\tau_{1}=0.31 \mathrm{~ns}(28 \%), \tau_{2}=1.72 \mathrm{~ns}(39 \%)$, and $\tau_{3}=$ $5.24 \mathrm{~ns}(33 \%)$ (corresponding to an average lifetime of 4.12 ns). Both the average lifetime and the lifetime components are very similar to those obtained for the tryptophan-flanked

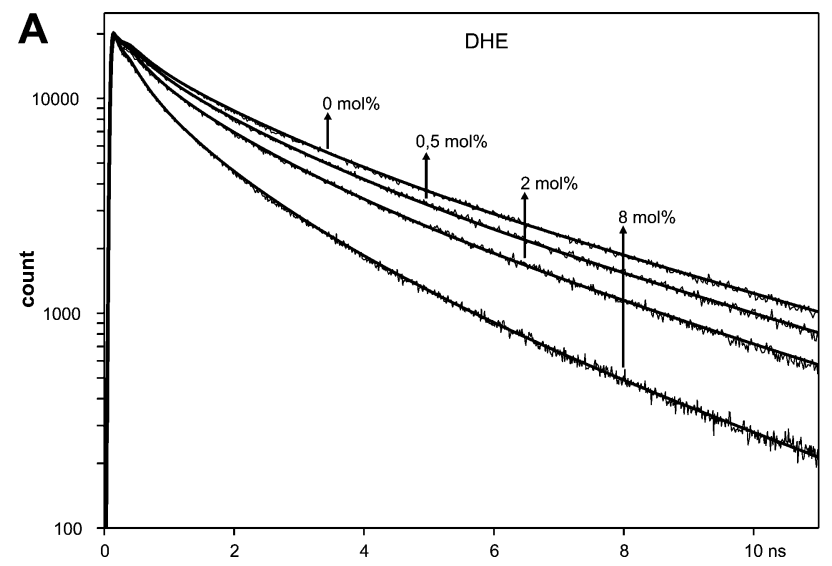

$\gamma \mathrm{M} 4$ peptide, an $\alpha$-helical transmembrane segment of the nicotinic acetylcholine receptor $(40,42)$.

In a global analysis approach, the decay curves for different concentrations of DHE (shown in Figure 4A) were fitted simultaneously on the basis of a FRET model for a single discrete concentration of acceptors according to eq 4 . With the exception of the varying acceptor density $n$, all other parameters $\left(R_{0}, R_{\mathrm{e}},\langle\tau\rangle\right.$, and $\left.w\right)$ were linked in the global analysis and therefore forced to have identical values for all decays, i.e., the same values as for the integrated curve with an $R_{0}$ of $20 \AA$ shown in Figure 3. Good quality fits from global analysis are characterized by a low global $\chi_{\mathrm{G}}{ }^{2}$, which is indicative of a good agreement with the underlying model of a random distribution of acceptor molecules in the bilayer. The global analysis of the decay series for bilayers containing WALP23 and different concentrations of DHE yielded a low $\chi_{\mathrm{G}}{ }^{2}$ of 1.43 which indicates a homogeneous distribution of the acceptor DHE in the bilayer (compare to ref 42). This result confirms the findings from steady-state fluorescence experiments.

Since the complex ring system of DHE has a threedimensional shape different from that of cholesterol, it is in principle possible that cholesterol, but not DHE, interacts preferentially with the tryptophan-flanked peptide. This question was addressed by a competition experiment where also cholesterol is present in the bilayer. If cholesterol would also be randomly distributed in the bilayer, the FRET efficiency should stay unchanged, whereas if cholesterol is attracted toward the tryptophans, it pushes the acceptor DHE away from the tryptophans and lowers the effective acceptor surface density in the vicinity of the donor, which in turn lowers the FRET efficiency (see Figure 5).

Steady-state fluorescence spectra were recorded for samples containing equimolar concentrations of DHE and cholesterol in the lipid fraction (Figure 2C), and the spectra were analyzed as described above. The results show that also in the presence of cholesterol the experimentally determined FRET efficiencies match the theoretical FRET efficiency curve for the Förster distance of WALP23 and DHE $(\triangle$ in Figure 3). Thus, the competition experiments indicate a random distribution of cholesterol in the bilayer. This was supported by fluorescence decay measurements on samples containing equimolar amounts of DHE and cholesterol in the lipid fraction (Figure 4B). There is no significant change

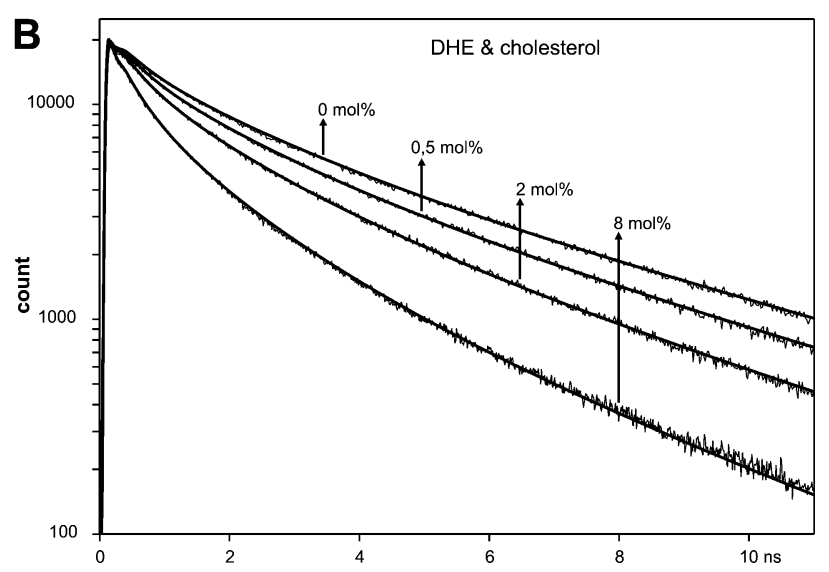

FIGURE 4: Fluorescence intensity decays of WALP23 in the presence of varying concentrations of DHE in bilayers of DMPC at pH 7.0 and $30{ }^{\circ} \mathrm{C}$. The bilayers contained either no cholesterol (A) or equimolar amounts of cholesterol (B). Global analysis of the data according to the model for a random distribution of DHE (eqs 2 and 3) resulted in the fitting curves shown yielding global $\chi_{\mathrm{G}}^{2}$ values of 1.43 for panel $\mathrm{A}$ and 1.35 for panel B. 

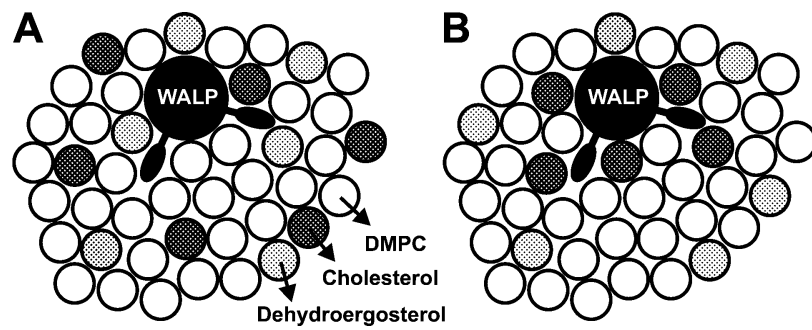

FiguRE 5: Schematic top view of the membrane illustrating two possible outcomes for the competition experiment using bilayers containing DHE and cholesterol. (A) Both DHE (gray circles) and cholesterol (black circles) are randomly distributed which leaves the FRET effciency unchanged when compared to that from the experiment in the absence of cholesterol. (B) Cholesterol occupies the sites next to the tryptophans of WALP23 and pushes in turn DHE away which leads to a lower FRET effciency.

in decay behavior if cholesterol is also added to the bilayer compared to bilayers containing only DHE (Figure 4A). Global analysis of the decay series for different equimolar concentrations of DHE and cholesterol yielded a $\chi_{\mathrm{G}}{ }^{2}$ of 1.35 , indicating a good agreement with the underlying model of random distribution of DHE (and therefore also of cholesterol) in the bilayer which strongly supports the finding of a random distribution of both DHE and cholesterol in the bilayer.

${ }^{2} \mathrm{H}$ NMR Results. All results obtained from fluorescence experiments indicate a random distribution of cholesterol in the bilayer in the presence of tryptophan-flanked peptides. Nevertheless, it is still possible that specific interactions occur between cholesterol and interfacial tryptophans which could influence the behavior of these molecules and thereby influence the properties of the membrane. Such an interaction could also be mediated via neighboring lipids. To investigate these possibilities, we performed ${ }^{2} \mathrm{H}$ NMR experiments on samples containing either deuterated cholesterol or tryptophan-deuterated WALP peptides. ${ }^{2} \mathrm{H}$ NMR spectroscopy is a very sensitive tool for observing small changes in dynamics and/or orientational properties of molecules and does not rely on the use of a fluorescent cholesterol analogue. Any change in orientation and/or dynamics of labeled molecules leads to a change in ${ }^{2} \mathrm{H}$ NMR spectra since the average orientation of the $\mathrm{C}-\mathrm{D}$ bonds with respect to the magnetic field is altered. ${ }^{2} \mathrm{H}$ NMR experiments can also be used to detect different populations of molecules that may occur if only parts of the population are influenced by specific interactions with other molecules. We investigated both the effect of WALP23 on deuterated cholesterol and the effect of cholesterol on tryptophan-deuterated WALP23.

Influence of Tryptophan-Flanked Peptides on Deuterated Cholesterol. For the ${ }^{2} \mathrm{H}$ NMR measurements, we used $\beta$ - $\left(d_{6}-\right.$ $2,2,3,4,4,6)$ cholesterol which has deuterons located near the hydroxyl headgroup. To investigate the influence of WALP23 peptides on deuterated cholesterol, we incorporated 2 or 4 mol \% cholesterol into unoriented DMPC bilayers and compared the spectra in the presence and absence of $1 \mathrm{~mol}$ $\%$ tryptophan-flanked WALP23 peptide. To distinguish effects due to the presence of interfacial tryptophans from effects due to the incorporation of peptides into the cholesterolcontaining bilayers, we used the lysine-flanked KALP23 peptide as a control peptide. At first glance, the ${ }^{2} \mathrm{H}$ NMR spectra of all these samples (shown in Figure 6) look very similar, indicating that there are no large effects on the

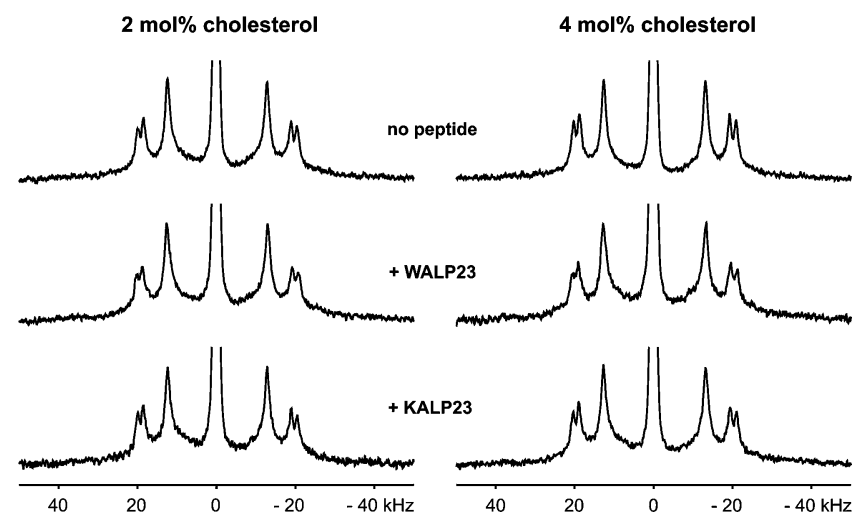

FIGURE 6: ${ }^{2} \mathrm{H}$ NMR spectra of $\beta-\left(d_{6}-2,2,3,4,4,6\right)$ cholesterol in unoriented bilayers of DMPC at $\mathrm{pH} 7.0$ and $40{ }^{\circ} \mathrm{C}$ in the absence and presence of KALP23 or WALP23. The quadrupolar splittings can be assigned to the deuterons in $\beta\left(d_{6}-2,2,3,4,4,6\right)$ cholesterol starting from outside: $(3),(2,4)_{\mathrm{ax}},(2,4)_{\mathrm{eq}}$; position (6) is hidden in the central isotropic peak $(43,57)$.

Table 2: Quadrupolar Splittings from ${ }^{2} \mathrm{H}$ NMR Spectra of Unoriented Samples Containing Deuterated Cholesterol

\begin{tabular}{lcccc}
\hline & & \multicolumn{3}{c}{ quadrupolar splittings $(\mathrm{kHz})$} \\
\cline { 3 - 5 } peptide & cholesterol $(\mathrm{mol} \%)$ & $(3)$ & $(2,4)_{\mathrm{ax}}$ & $(2,4)_{\mathrm{eq}}$ \\
\hline- & 2 & 40.4 & 37.4 & 25.2 \\
& 4 & 41.2 & 38.1 & 25.8 \\
WALP23 & 2 & 41.0 & 38.0 & 25.6 \\
& 4 & 41.8 & 38.7 & 26.2 \\
KALP23 & 2 & 40.4 & 37.5 & 25.2 \\
& 4 & 41.3 & 38.3 & 25.9 \\
WLP23 & 2 & 42.3 & 39.4 & 26.5 \\
KLP23 & 2 & 40.7 & 37.8 & 25.4 \\
\hline
\end{tabular}

orientation and dynamics of cholesterol. However, some subtle but significant changes in the values of the quadrupolar splittings occur which will be discussed later.

The ${ }^{2} \mathrm{H}$ NMR spectrum for a bilayer containing $2 \mathrm{~mol} \%$ deuterated cholesterol in the absence of peptide (top row in Figure 6) is typical for $\beta$ - $\left(d_{6}-2,2,3,4,4,6\right)$ cholesterol incorporated into fluid phase bilayers (43). Knowing that the orientation of cholesterol in the bilayer is rather fixed along its long axis, the similarity of the ${ }^{2} \mathrm{H}$ NMR spectra suggests the same assignment of the splittings to the deuterons in all cases which allows direct comparison of the quadrupolar splittings summarized in Table 2.

Closer inspection of the quadrupolar splittings in the absence of peptides shows that addition of more cholesterol (4 mol \%) leads to a small increase of $0.6-0.8 \mathrm{kHz}$ in quadrupolar splittings. Cholesterol is known to straighten the acyl chains of surrounding lipids which leads to a condensation effect on the membrane bilayer. This affects the dynamics of cholesterol itself which leads to the larger quadrupolar splittings (43). This observation demonstrates that ${ }^{2} \mathrm{H}$ NMR spectroscopy employing deuterated cholesterol is a sensitive tool for observing very small changes in the behavior of cholesterol.

The presence of WALP23 peptides in the bilayer leads to a small but significant increase of $0.4-0.6 \mathrm{kHz}$ in the quadrupolar splittings at both cholesterol concentrations that were used. Control experiments using $1 \mathrm{~mol} \%$ KALP23 show no significant increase in the quadrupolar splittings in the presence of a lysine-flanked peptide. This could suggest an interaction between cholesterol and tryptophan. However, it was shown before that WALP23 also has a stretching effect 
on the bilayer similar to that of cholesterol (18) which could cause an increase in quadrupolar splittings. Therefore, the increase in quadrupolar splittings could also be due to the membrane stretching effect of WALP23.

To discriminate between these possibilities, we investigated how membrane stretching due to the presence of peptides in the membrane influences the quadrupolar splittings. For this purpose, we used WLP23 and KLP23 which have the same length as WALP23 and KALP23 but have a more hydrophobic stretch of only leucines. Previously, it was found that a lysine-flanked transmembrane peptide having a hydrophobic core of polyleucines exerts a stronger ordering effect on lipid acyl chains than an analogous peptide with a core of alternating leucines and alanines (44). Preliminary experiments in our laboratory indicated that this is also the case for WLP23 and KLP23. If membrane stretching due to the peptides in the bilayer would be the only factor responsible for the increase in quadrupolar splittings of deuterated cholesterol, then we should expect an increase in the quadrupolar splittings for WLP23 compared to WALP23 and for KLP23 compared to KALP23. Evaluation of the quadrupolar splittings for WLP23 and KLP23 listed in Table $2\left({ }^{2} \mathrm{H}\right.$ NMR spectra not shown) shows that the presence of the polyleucine peptides in the membrane indeed leads to a significant increase in the quadrupolar splittings as compared to those of the leucine-alanine peptides. Thus, the difference in the quadrupolar splittings in the presence of WALP23 and KALP23 in the bilayer can most likely be attributed to differences in the stretching effect of the peptides.

It is important to note that all ${ }^{2} \mathrm{H}$ NMR spectra compared show only one component upon addition of WALP23 peptides to the bilayer. This absence of a second component points toward one major population of cholesterol in the bilayer. Therefore, the ${ }^{2} \mathrm{H}$ NMR experiments using deuterated cholesterol provide no indication for an effect on the dynamics and/or orientation of cholesterol caused by an interaction with interfacial tryptophans.

Influence of Cholesterol on Deuterated Tryptophan Side Chains. Next, in a complementary approach, we investigated whether the presence of cholesterol changes the orientation and/or dynamics of the interfacial tryptophans. We performed ${ }^{2} \mathrm{H}$ NMR experiments using tryptophan-deuterated WALP23 peptides. The peptides were synthesized with tryptophans containing a deuterated indole ring in one position at a time (for amino acid sequences, see Table 1). Oriented samples with a peptide/lipid ratio of $1 / 25$ containing either no or $4 \mathrm{~mol} \%$ cholesterol in the lipid fraction were prepared. ${ }^{31} \mathrm{P}$ NMR measurements on all oriented samples confirmed a good alignment of the bilayers along the glass platelets ${ }^{31} \mathrm{P}$ NMR spectra not shown). The ${ }^{2} \mathrm{H}$ NMR spectra of samples containing WALP23- $d_{5}$-Trp2 and WALP23- $d_{5}$-Trp3 in the presence and absence of cholesterol are shown in Figure 7. The measurements were performed at two different orientations of the bilayers: at $0^{\circ}$ with the membrane normal aligned to the magnetic field and at $90^{\circ}$ with the membrane normal perpendicular to the magnetic field.

The deuterated indole ring contains five deuterons where two deuterons (positions 4 and 7) are equivalent in view of the geometry of the indole ring and therefore bear the same quadrupolar splitting (21). Thus, four quadrupolar splittings are expected for WALP23 with indole ring-deuterated tryptophans. Previous ${ }^{2} \mathrm{H}$ NMR experiments on oriented
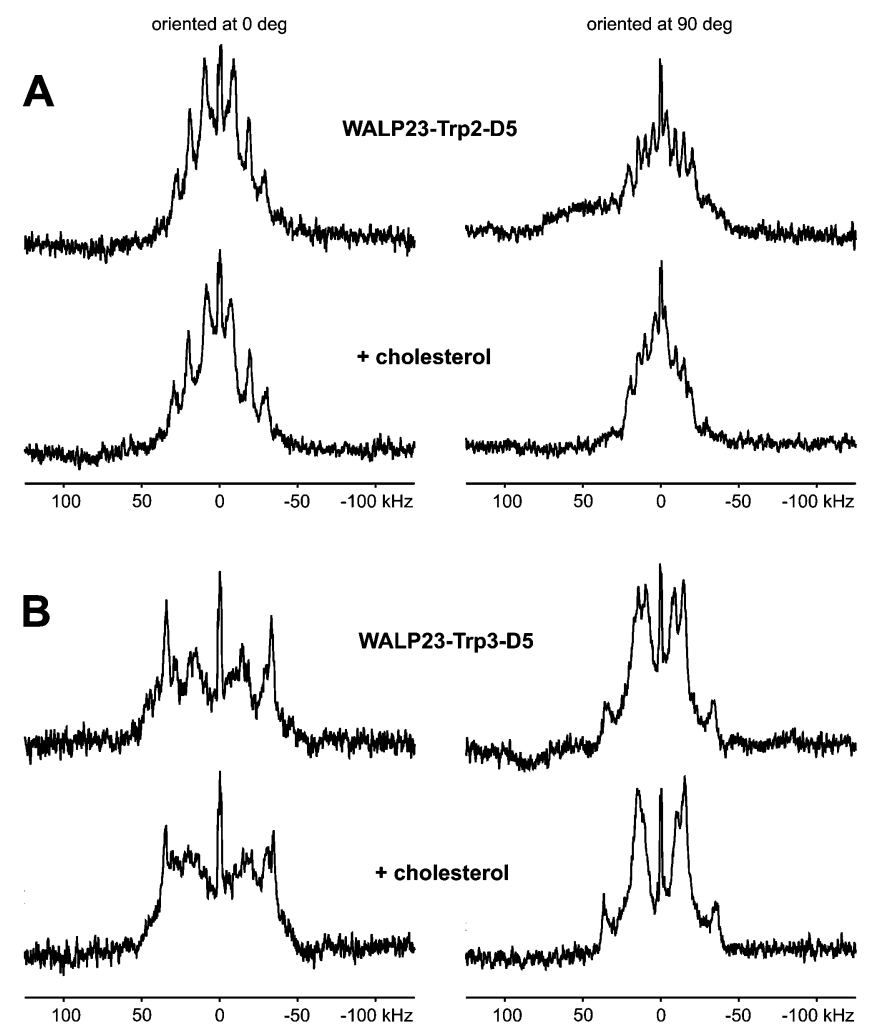

FIGURE 7: ${ }^{2} \mathrm{H}$ NMR spectra of WALP23- $d_{5}$-Trp2 (A) and WALP23 $d_{5}$-Trp3 (B) in oriented bilayers of DMPC at pH 7.0 in the absence and presence of $4 \mathrm{~mol} \%$ cholesterol added to the lipid fraction at $40 \%(\mathrm{w} / \mathrm{w})$ hydration and measured at $40{ }^{\circ} \mathrm{C}$.

Table 3: Quadrupolar Splittings from ${ }^{2} \mathrm{H}$ NMR Spectra of Oriented Samples Containing WALP23- $d_{5}$-Trp2 in the Absence and Presence of Cholesterol

\begin{tabular}{lcrccc}
\hline & cholesterol $(\mathrm{mol} \%)$ & \multicolumn{4}{c}{ quadrupolar splittings $^{a}(\mathrm{kHz})$} \\
\hline $0^{\circ}$ & - & 18 & 38 & 57 & - \\
$90^{\circ}$ & - & 9 & 19 & 29 & 41 \\
$0^{\circ}$ & 4 & 15 & 40 & 60 & - \\
$90^{\circ}$ & 4 & 6 & 20 & 30 & 39 \\
\hline
\end{tabular}

${ }^{a}$ The quadrupolar splittings are estimated to have an experimental error of $1-2 \mathrm{kHz}$ (compare to ref 48 ).

samples containing the tryptophan-flanked gramicidin A (21, $45,46)$ or WALP peptides $(47,48)$ showed that not all splittings are always detected for either orientation of the bilayers with respect to the magnetic field. Looking at the ${ }^{2} \mathrm{H}$ NMR spectra for WALP23- $d_{5}$-Trp2, we can distinguish four splittings for the $90^{\circ}$ orientation (Figure 7A, right column) compared to only three splittings for the $0^{\circ}$ orientation (Figure 7A, left column). The size of the quadrupolar splittings in the $90^{\circ}$ orientation can be expected to be halved compared to the $0^{\circ}$ orientation due to the fast axial reorientation of the peptides about the bilayer normal (36). Comparison of the values for WALP23- $d_{5}$-Trp2 in Table 3 shows that this is indeed the case. From these values, it can furthermore be concluded that the largest splitting in the $0^{\circ}$ orientation is not detected. This is probably due to insufficient sensitivity caused by the magnitude of the quadrupolar splittings in combination with the relatively low peptide/lipid ratio.

The assignments of the quadrupolar splittings to the deuterons of the indole ring cannot be done unambiguously without further experiments, e.g., ${ }^{2} \mathrm{H}$ NMR experiments with 
partly deuterated indole rings (21) or NMR experiments employing other labels. Nevertheless, the quadrupolar splittings can be considered as fingerprints for the orientational and motional properties of the tryptophan side chains. The shape of the ${ }^{2} \mathrm{H}$ NMR spectrum of WALP23- $d_{5}$-Trp2 does not change upon addition of cholesterol (compare the top and bottom rows of Figure 7A), and there is no significant increase or decrease in the visible quadrupolar splittings (see Table 3). For WALP23- $d_{5}$-Trp3, it is more difficult to distinguish (all) the quadrupolar splittings (data not listed), but also for this peptide, the same findings hold (Figure 7B). The results therefore suggest that the orientation and dynamics of the indole rings are not influenced by cholesterol (on the ${ }^{2} \mathrm{H}$ NMR time scale). Hence, these experiments also indicate that there is no specific interaction between cholesterol and interfacial tryptophans.

\section{DISCUSSION}

The main objective of this study was to investigate how cholesterol and interfacial tryptophans of transmembrane peptides or proteins interact in a bilayer and what the possible consequences are for the organization and distribution of these molecules in membranes. For our study, we chose a well-defined model system of tryptophan-flanked transmembrane peptides incorporated into cholesterol-containing bilayers, sampling a biologically relevant system for such an interaction. To gain insight into the interaction between cholesterol and interfacial tryptophans, we employed a 2-fold approach, including FRET experiments and solid-state ${ }^{2} \mathrm{H}$ NMR measurements.

Choice for a Low Cholesterol Concentration in the Bilayer. All experiments were performed on bilayers containing only small amounts of cholesterol in the lipid fraction. One reason for this was the consideration that if indeed preferential interactions would occur, they would most easily be observed at low cholesterol concentrations where random contacts between the peptide and the sterol are only few in number. Even more important, however, was the consideration that a low cholesterol content is essential to ensure a homogeneous system. It is well-known that higher cholesterol concentrations in a phospholipid bilayer can lead to the formation of coexistent phases in the bilayer $(49,50)$. The onset of phase separation into liquid-ordered $\left(\mathrm{L}_{\mathrm{o}}\right)$ and liquiddisordered $\left(\mathrm{L}_{\mathrm{d}}\right)$ domains is strongly dependent on temperature and cholesterol content. For the two-component system used in this study (DMPC/cholesterol), at $30{ }^{\circ} \mathrm{C}$ the phase separation starts at $8 \mathrm{~mol} \%$ cholesterol in the bilayer and at $40{ }^{\circ} \mathrm{C}$ at $14 \mathrm{~mol} \%$ cholesterol (51), generating a laterally heterogeneous composition of the bilayer. The liquid-ordered domains contain a higher cholesterol concentration and have an increased membrane thickness compared to the liquiddisordered domains. The resulting heterogeneous system would complicate, if not prohibit, the interpretation of the experimental data.

This holds in particular for the FRET experiments where data analysis is based on the comparison of experimental FRET efficiencies with theoretical FRET efficiencies where the values are calculated on the basis of a random distribution of donor and acceptor in the bilayer. In the case of a phase separation in the bilayer, more cumbersome models would have to be used, which would have to account, among other complexities, for the partitioning of both peptide and DHE between the different phases (41). For these various reasons, we decided to use only small cholesterol concentrations of $<8 \mathrm{~mol} \%$ cholesterol in DMPC bilayers for our experiments.

Cholesterol Does Not Preferentially Localize Next to Tryptophan-Flanked Peptides. In case of a preferential interaction with interfacial tryptophans, one would expect a nonrandom distribution of cholesterol in the bilayer when a tryptophan-flanked peptide is included. The sterol would be preferentially localized in the vicinity of the peptide and hence would be depleted from the bulk lipid. To analyze the distribution of cholesterol in the bilayer, FRET experiments were performed in which we used the tryptophans of the WALP23 peptides as the donor and the fluorescent cholesterol analogue DHE as the acceptor. We found that DHE is randomly distributed in the presence of interfacial tryptophans, suggesting that there is no preferential interaction between DHE and interfacial tryptophans.

In these experiments, DHE was used because cholesterol does not possess a chromophore with absorption overlapping the emission of tryptophan and because DHE was shown to be suitable for investigation of sterol behavior in the membrane (25). Similar studies employing the cholesterol analogue DHE as the acceptor for FRET experiments have been conducted with, for example, melittin (52) and the $\gamma \mathrm{M} 4$ segment of the nicotinic acetylcholine receptor (AChR) (40). Although cholestatrienol, another fluorescent cholesterol analogue, mimics the properties of cholesterol in the membrane better (53), this analogue has some disadvantages compared to DHE. It degrades rapidly and requires diligent sample handling (54). With regard to the special properties of cholesterol in the membrane, both analogues do mimic cholesterol only to a limited extent and have poorer condensation effects on the bilayer $(53,55)$. This emerges from two extra double bonds in the complex ring structure of DHE and cholestatrienol, which cause the fluorescent character but also change the three-dimensional shape of the analogues in comparison to that of cholesterol.

In principle, it is possible that the difference in threedimensional structure between DHE and cholesterol could change the behavior with respect to a possible (stacking) interaction with interfacial tryptophans. To gain information about the distribution of cholesterol itself in bilayers containing tryptophan-flanked peptides, we performed a competition experiment in which both the fluorescent cholesterol analogue DHE and cholesterol were incorporated into the bilayers at the same concentration. Cholesterol was found not to compete with DHE for sites next to the tryptophans which strongly suggests that cholesterol is randomly distributed in the bilayer. This finding points toward no preferential interaction between cholesterol and interfacial tryptophans, suggesting that it is equally favorable for cholesterol to be adjacent to WALP23 peptides as it is to be in contact with the lipid acyl chains.

Cholesterol and Interfacial Tryptophans Do Not Affect Each Other's Orientation and/or Dynamics. Even for a random distribution of cholesterol in the bilayer it is still possible that cholesterol and interfacial tryptophans affect each other in a specific way, possibly mediated by adjacent lipids. Since any interaction between two molecules is associated with changes in the dynamics and/or orientation of the molecules involved, we used solid-state ${ }^{2} \mathrm{H}$ NMR 
spectroscopy to test this hypothesis. This technique is a sensitive tool for observing small changes in dynamics and/or orientation of deuterium-labeled compounds in a lipid bilayer.

We performed ${ }^{2} \mathrm{H}$ NMR measurements employing either deuterated cholesterol or tryptophan-deuterated WALP23 peptides. We found that the magnitudes of the quadrupolar splittings yielded by the deuterated tryptophans do not change after addition of cholesterol to the bilayer. In the case of deuterated cholesterol, small changes in the sizes of the quadrupolar splittings were observed which could be attributed to small differences in the effects of the peptides on lipid chain order in the bilayer. These findings suggest that there is no change in the average orientation or in dynamics for either cholesterol or the tryptophan side chains of WALP23 and therefore that these molecules have neither a direct nor a lipid-mediated influence on each other. Another important observation was that for all ${ }^{2} \mathrm{H}$ NMR spectra recorded in the presence of either deuterated cholesterol or deuterated tryptophan-flanked peptides in the bilayer, only one component could be distinguished. This absence of a second population is another indication of the absence of specific interactions resulting in preferential surrounding of the peptide by cholesterol. Hence, these findings are in good agreement with the results from the FRET experiments.

Nature of Interaction between Cholesterol and Interfacial Tryptophans. Via examination of the molecular structures of cholesterol and tryptophan, it is very well conceivable that the complex ring structure of cholesterol directly interacts with the tryptophan indole ring. Indeed, molecular modeling studies suggested favorable stacking interactions between cholesterol and tryptophan where the face of the complex ring system of cholesterol and the indole ring of tryptophan build the interaction interface (personal communication with R. Brasseur, CBMN, Gembloux, Belgium). In a phospholipid bilayer, the long axis and therefore also the flat face of the complex ring system of cholesterol are oriented almost parallel to the bilayer normal $(56,57)$. It is possible to calculate the orientation of the tryptophan side chain with respect to the bilayer from the quadrupolar splittings of a deuterated indole ring if the spectral assignments are known $(21,46)$.

Preliminary attempts to calculate the orientations of the deuterated tryptophans of WALP23- $d_{5}$-Trp2 and WALP23$d_{5}$-Trp3 using an automatic assignment procedure yielded for both tryptophan positions a reliable minimum for a single orientation. The best fits to the sets of quadrupolar splittings summarized in Table 3 yielded very similar orientations both in the absence and in the presence of cholesterol in the bilayer. For both WALP23- $d_{5}-\operatorname{Trp} 2$ and WALP23- $d_{5}$-Trp3, the flat face of the tryptophan indole ring adopts an angle of approximately $30^{\circ}$ with respect to the bilayer normal which is comparable to the orientations determined for the tryptophan side chains in gramicidin A (21). Such an orientation of the tryptophan indole ring would imply that for a stacking interaction either the indole ring or the cholesterol would need to turn $\sim 30^{\circ}$ with respect to the bilayer normal. ${ }^{2} \mathrm{H}$ NMR experiments clearly show that the orientations of both the indole ring and cholesterol remain unchanged in the presence of the respective other molecule in the bilayer. In view of these considerations, this suggests that no direct stacking interaction occurs between tryptophan and cholesterol.
In general, two molecules will preferentially interact if this interaction leads to a decrease in the free energy of the system. Apparently, the energy cost for changing the orientation of interfacial tryptophans and/or cholesterol to facilitate a preferential interaction in combination with the loss of entropy resulting from such an interaction is higher than the energy gain from a specific interaction between the two molecules. By itself, this is not surprising. The orientation of cholesterol in the bilayer is governed by its amphiphilic properties whereby the small hydroxyl headgroup of cholesterol preferably resides between the phospholipid headgroups. A change in the orientation of cholesterol, e.g., of $30^{\circ}$ with respect to the bilayer normal, can be expected to result in a large energy cost due to its preferable alignment alongside the lipid acyl chains. The side chain of tryptophan is small compared to cholesterol, but it is connected to a larger peptide backbone, which restricts the number of possible and energetically favorable orientations. Statistical analysis of side chain conformations in $\alpha$-helices of known structures of (membrane) proteins shows that only certain combinations of $\phi$ and $\psi$ torsion angles for tryptophans are feasible and favorable $(58,59)$. These constraints may prevent a preferential interaction between tryptophans and cholesterol in the bilayer.

The results of this study seem in contradiction with all earlier indications found for a specific interaction between cholesterol and tryptophans located in the bilayer. How can we explain this? It is important to note here that all experiments indicating such a preferential interaction were carried out in bilayer systems with a relatively high cholesterol content. Either the protein resides in a membrane that (natively) has a very high cholesterol content, as is the case of the acetylcholine receptor-enriched membranes from the neuro-muscular junction (14), or the membrane contains cholesterol-rich domains, as for caveolae (13) or lipid rafts (1), into which the protein partitions favorably. High cholesterol concentrations in a bilayer change the behavior of the cholesterol in the bilayer itself as well as the material properties of the bilayers compared to a bilayer containing no or only low concentrations of cholesterol (60). Thus, it is possible that the two situations may not be comparable and that our findings may not be applicable to proteins in caveolae and rafts. Furthermore, in the case in which a tryptophan-containing peptide or protein is present in a cholesterol-rich environment, this will increase the probability for their interaction. Together with the changed bilayer properties, this may result in a modulation of membrane and protein structure and dynamics which in the studies mentioned above has been thought to possibly involve a preferential interaction between cholesterol and tryptophans.

The suggested specificity for cholesterol and tryptophan in these studies may also be related to the anchoring properties of tryptophans in membrane proteins. Previously, it has been observed that tryptophans are preferably located in the lipid-water interface $(7,9,61)$. Higher cholesterol concentrations are known to lead to a laterally heterogeneous bilayer containing cholesterol-enriched domains which have an increased thickness compared to the cholesterol-depleted domains. Depending on its hydrophobic length, the peptide or protein can be driven toward the thicker and thus cholesterol-enriched domains of the bilayer to relieve the hydrophobic mismatch. Since tryptophans have been shown 
to be essential for the proper positioning of proteins in (biological) membranes $(62-65)$, a mutation in this residue may place it out of cholesterol-rich domains even when there is no specific interaction between cholesterol and interfacial tryptophans. Hence, there would be only an apparent specificity.

In any case, our study on model systems with relatively low cholesterol concentrations has clearly demonstrated that cholesterol and tryptophan do not have a general tendency to preferentially interact with one another. This is an important result, because it improves our understanding of the basic principles behind the organization of proteins and lipids in membranes, here in particular between interfacial tryptophans abundant in membrane proteins and cholesterol, a membrane constituent in many types of biological membranes.

\section{ACKNOWLEDGMENT}

We thank R. Brasseur, A. Thomas (Centre de Biophysique Moléculaire Numérique, Faculté Universitaire des Sciences Agronomiques de Gembloux, Gembloux, Belgium), and B. de Kruijff (Utrecht University) for stimulating discussions and A. Fedorov (Instituto Superior Técnico) for help with the time-resolved fluorescence spectroscopy measurements.

\section{REFERENCES}

1. Epand, R. M. (2006) Cholesterol and the interaction of proteins with membrane domains, Prog. Lipid Res. 45, 279-294.

2. Pike, L. J. (2004) Lipid rafts: Heterogeneity on the high seas, Biochem. J. 378, 281-292.

3. Hancock, J. F. (2006) Lipid rafts: Contentious only from simplistic standpoints, Nat. Rev. Mol. Cell Biol. 7, 456-462.

4. Ohvo-Rekilä, H., Ramstedt, B., Leppimäki, P., and Slotte, J. P. (2002) Cholesterol interactions with phospholipids in membranes, Prog. Lipid Res. 41, 66-97.

5. van Duyl, B. Y., Meeldijk, H., Verkleij, A. J., Rijkers, D. T. S., Chupin, V., de Kruijff, B., and Killian, J. A. (2005) A synergistic effect between cholesterol and tryptophan-flanked transmembrane helices modulates membrane curvature, Biochemistry 44, 45264532.

6. Yau, W.-M., Wimley, W. C., Gawrisch, K., and White, S. H. (1998) The preference of tryptophan for membrane interfaces, Biochemistry 37, 14713-14718.

7. Schiffer, M., Chang, C. H., and Stevens, F. J. (1992) The functions of tryptophan residues in membrane proteins, Protein Eng. 5, 213-214.

8. Braun, P., and von Heijne, G. (1999) The aromatic residues Trp and Phe have different effects on the positioning of a transmembrane helix in the microsomal membrane, Biochemistry 38, 97789782.

9. de Planque, M. R. R., Bonev, B. B., Demmers, J. A. A., Greathouse, D. V., Koeppe, R. E., II, Separovic, F., Watts, A., and Killian, J. A. (2003) Interfacial anchor properties of tryptophan residues in transmembrane peptides can dominate over hydrophobic matching effects in peptide-lipid interactions, Biochemistry $42,5341-5348$.

10. White, S. H., and Wimley, W. C. (1998) Hydrophobic interactions of peptides with membrane interfaces, Biochim. Biophys. Acta $1376,339-352$.

11. Dougherty, D. A. (1996) Cation- $\pi$ interactions in chemistry and biology: A new view of benzene, Phe, Tyr, and Trp, Science 271, 163-168.

12. Gasset, M., Killian, J. A., Tournois, H., and de Kruijff, B. (1988) Influence of cholesterol on gramicidin-induced HII phase formation in phosphatidylcholine model membranes, Biochim. Biophys. Acta 939, 79-88.

13. Carozzi, A. J., Roy, S., Morrow, I. C., Pol, A., Wyse, B., ClydeSmith, J., Prior, I. A., Nixon, S. J., Hancock, J. F., and Parton, R. G. (2002) Inhibition of lipid raft-dependent signaling by a dystrophy-associated mutant of caveolin-3, J. Biol. Chem. 277, 17944-17949.
14. Santiago, J., Guzmán, G. R., Rojas, L. V., Marti, R., Asmar-Rovira, G. A., Santana, L. F., McNamee, M., and Lasalde-Dominicci, J. A. (2001) Probing the effects of membrane cholesterol in the Torpedo californica acetylcholine receptor and the novel lipidexposed mutation $\alpha \mathrm{C} 418 \mathrm{~W}$ in Xenopus oocytes, J. Biol. Chem. $276,46523-46532$.

15. Killian, J. A., and von Heijne, G. (2000) How proteins adapt to a membrane-water interface, Trends Biochem. Sci. 25, 429-434.

16. Killian, J. A. (2003) Synthetic peptides as models for intrinsic membrane proteins, FEBS Lett. 555, 134-138.

17. Killian, J. A., and Nyholm, T. K. M. (2006) Peptides in lipid bilayers: The power of simple models, Curr. Opin. Struct. Biol. $16,473-479$

18. de Planque, M. R. R., Kruijtzer, J. A. W., Liskamp, R. M. J., Marsh, D., Greathouse, D. V., Koeppe, R. E., II, de Kruijff, B., and Killian, J. A. (1999) Different membrane anchoring positions of tryptophan and lysine in synthetic transmembrane $\alpha$-helical peptides, J. Biol. Chem. 274, 20839-20846.

19. ten Kortenaar, P. B. W., van Dijk, B. G., Peeters, J. M., Raaben, B. J., Adams, P. J. H. M., and Tesser, G. I. (1986) Rapid and efficient method for the preparation of Fmoc-amino acids starting from 9-fluorenylmethanol, Int. J. Pept. Protein Res. 27, 398400

20. Greathouse, D. V., Koeppe, R. E., II, Providence, L. L., Shobana, S., and Andersen, O. S. (1999) Design and characterization of gramicidin channels, Methods Enzymol. 294, 525-550.

21. Koeppe, R. E., II, Sun, H., van der Wel, P. C. A., Scherer, E. M., Pulay, P., and Greathouse, D. V. (2003) Combined experimental/ theoretical refinement of indole ring geometry using deuterium magnetic resonance and ab initio calculations, J. Am. Chem. Soc. $125,12268-12276$.

22. Rijkers, D. T. S., Kruijtzer, J. A. W., Killian, J. A., and Liskamp, R. M. J. (2005) A convenient solid phase synthesis of S-palmitoyl transmembrane peptides, Tetrahedron Lett. 46, 3341-3345.

23. Rouser, G., Fleischer, S., and Yamamoto, A. (1970) Twodimensional thin layer chromatographic separation of polar lipids and determination of phospholipids by phosphorus analysis of spots, Lipids 5, 494-496.

24. Sparr, E., Ganchev, D. N., Snel, M. M. E., Ridder, A. N. J. A., Kroon-Batenburg, L. M. J., Chupin, V., Rijkers, D. T. S., Killian, J. A., and de Kruijff, B. (2005) Molecular organization in striated domains induced by transmembrane $\alpha$-helical peptides in dipalmitoyl phosphatidylcholine bilayers, Biochemistry 44, 2-10.

25. Smutzer, G., Crawford, B. F., and Yeagle, P. L. (1986) Physical properties of the fluorescent sterol probe dehydroergosterol, Biochim. Biophys. Acta 862, 361-371.

26. Gardecki, J. A., and Maroncelli, M. (1998) Set of secondary emission standards for calibration of the spectral responsivity in emission spectroscopy, Appl. Spectrosc. 52, 1179-1189.

27. Loura, L. M. S., Fedorov, A., and Prieto, M. (1996) Resonance energy transfer in a model system of membranes: Application to gel and liquid crystalline phases, Biophys. J. 71, 1823-1836.

28. Loura, L. M. S., Fedorov, A., and Prieto, M. (2000) Membrane probe distribution heterogeneity: A resonance energy transfer study, J. Phys. Chem. B 104, 6920-6931.

29. Marquardt, D. W. (1963) An algorithm for least-squares estimation of nonlinear parameters, J. Soc. Ind. Appl. Math. 11, 431-441.

30. Lakowicz, J. R. (1999) Principles of Fluorescence Spectroscopy, Kluwer Academic/Plenum Publishers, New York.

31. Davenport, L., Dale, R. E., Bisby, R. H., and Cundall, R. B. (1985) Transverse location of the fluorescent probe 1,6-diphenyl-1,3,5hexatriene in model lipid bilayer membrane systems by resonance excitation energy transfer, Biochemistry 24, 4097-4108.

32. Salamon, Z., Lindblom, G., Rilfors, L., Linde, K., and Tollin, G. (2000) Interaction of phosphatidylserine synthase from E. coli with lipid bilayers: Coupled plasmon-waveguide resonance spectroscopy studies, Biophys. J. 78, 1400-1412.

33. Stryer, L. (1978) Fluorescence energy transfer as a spectroscopic ruler, Annu. Rev. Biochem. 47, 819-846.

34. Vos, W. L., Koehorst, R. B. M., Spruijt, R. B., and Hemminga, M. A. (2005) Membrane-bound conformation of M13 major coat protein: A structure validation through FRET-derived constraints, J. Biol. Chem. 280, 38522-38527.

35. Wolber, P. K., and Hudson, B. S. (1979) An analytic solution to the Förster energy transfer problem in two dimensions, Biophys. J. 28, 197-210.

36. Strandberg, E., Özdirekcan, S., Rijkers, D. T. S., van der Wel, P. C. A., Koeppe, R. E., II, Liskamp, R. M. J., and Killian, J. A (2004) Tilt angles of transmembrane model peptides in oriented 
and non-oriented lipid bilayers as determined by ${ }^{2} \mathrm{H}$ solid-state NMR, Biophys. J. 86, 3709-3721.

37. Davis, J. H. (1983) The description of membrane lipid conformation, order and dynamics by ${ }^{2} \mathrm{H}-\mathrm{NMR}$, Biochim. Biophys. Acta 737, 117-171.

38. Koenig, B. W., Strey, H. H., and Gawrisch, K. (1997) Membrane lateral compressibility determined by NMR and X-ray diffraction: Effect of acyl chain polyunsaturation, Biophys. J. 73, 19541966.

39. Smaby, J. M., Momsen, M. M., Brockman, H. L., and Brown, R. E. (1997) Phosphatidylcholine acyl unsaturation modulates the decrease in interfacial elasticity induced by cholesterol, Biophys. J. 73, 1492-1505.

40. de Almeida, R. F. M., Loura, L. M. S., Prieto, M., Watts, A., Fedorov, A., and Barrantes, F. J. (2004) Cholesterol modulates the organization of the $\gamma \mathrm{M} 4$ transmembrane domain of the muscle nicotinic acetylcholine receptor, Biophys. J. 86, 2261-2272.

41. Loura, L. M. S., de Almeida, R. F. M., and Prieto, M. (2001) Detection and characterization of membrane microheterogeneity by resonance energy transfer, J. Fluoresc. 11, 197-209.

42. de Almeida, R. F. M., Loura, L. M. S., Prieto, M., Watts, A., Fedorov, A., and Barrantes, F. J. (2006) Structure and dynamics of the $\gamma \mathrm{M} 4$ transmembrane domain of the acetylcholine receptor in lipid bilayers: Insights into receptor assembly and function, Mol. Membr. Biol. 23, 305-315.

43. Léonard, A., and Dufourc, E. (1991) Interactions of cholesterol with the membrane lipid matrix. A solid state NMR approach, Biochimie 73, 1295-1302.

44. Paré, C., Lafleur, M., Liu, F., Lewis, R. N., and McElhaney, R. N. (2001) Differential scanning calorimetry and ${ }^{2} \mathrm{H}$ nuclear magnetic resonance and Fourier transform infrared spectroscopy studies of the effects of transmembrane $\alpha$-helical peptides on the organization of phosphatidylcholine bilayers, Biochim. Biophys. Acta 1511, 60-73.

45. Killian, J. A., Taylor, M. J., and Koeppe, R. E., II (1992) Orientation of the valine-1 side chain of the gramicidin transmembrane channel and implications for channel functioning. A ${ }^{2} \mathrm{H}$ NMR study, Biochemistry 31, 11283-11290.

46. Koeppe, R. E., II, Killian, J. A., and Greathouse, D. V. (1994) Orientations of the tryptophan 9 and 11 side chains of the gramicidin channel based on deuterium nuclear magnetic resonance spectroscopy, Biophys. J. 66, 14-24.

47. van der Wel, P. C. A., Strandberg, E., Killian, J. A., and Koeppe, R. E., II (2002) Geometry and intrinsic tilt of a tryptophananchored transmembrane $\alpha$-helix determined by ${ }^{2} \mathrm{H}$ NMR, Biophys. J. 83, 1479-1488.

48. van der Wel, P. C. A., Reed, N. D., Greathouse, D. V., and Koeppe, R. E., II (2007) Orientation and motion of tryptophan interfacial anchors in membrane-spanning peptides, Biochemistry 46, 75147524.

49. Ipsen, J. H., Mouritsen, O. G., and Bloom, M. (1990) Relationships between lipid membrane area, hydrophobic thickness, and acylchain orientational order: The effects of cholesterol, Biophys. J. $57,405-412$.

50. Silvius, J. R. (2003) Role of cholesterol in lipid raft formation: Lessons from lipid model systems, Biochim. Biophys. Acta 1610, 174-183.
51. Almeida, P. F. F., Vaz, W. L. C., and Thompson, T. E. (1992) Lateral diffusion in the liquid phases of dimyristoylphosphatidylcholine/cholesterol lipid bilayers: A free volume analysis, Biochemistry 31, 6739-6747.

52. Raghuraman, H., and Chattopadhyay, A. (2004) Interaction of melittin with membrane cholesterol: A fluorescence approach, Biophys. J. 87, 2419-2432.

53. Scheidt, H. A., Müller, P., Herrmann, A., and Huster, D. (2003) The potential of fluorescent and spin-labeled steroid analogs to mimic natural cholesterol, J. Biol. Chem. 278, 45563-45569.

54. Fischer, R. T., Stephenson, F. A., Shafiee, A., and Schroeder, F. (1984) $\Delta^{5,7,9(11)}$-Cholestatrien-3 $\beta$-ol: A fluorescent cholesterol analogue, Chem. Phys. Lipids 36, 1-14.

55. Ohvo-Rekilä, H., Åkerlund, B., and Slotte, J. P. (2000) Cyclodextrin-catalyzed extraction of fluorescent sterols from monolayer membranes and small unilamellar vesicles, Chem. Phys. Lipids 105, 167-178.

56. Dufourc, E. J., Parish, E. J., Chitrakorn, S., and Smith, I. C. P. (1984) Structural and dynamical details of cholesterol-lipid interaction as revealed by deuterium NMR, Biochemistry 23, 6062-6071.

57. Marsan, M. P., Muller, I., Ramos, C., Rodriguez, F., Dufourc, E. J., Czaplicki, J., and Milon, A. (1999) Cholesterol orientation and dynamics in dimyristoylphosphatidylcholine bilayers: A solid state deuterium NMR analysis, Biophys. J. 76, 351-359.

58. Chamberlain, A. K., and Bowie, J. U. (2004) Analysis of sidechain rotamers in transmembrane proteins, Biophys. J. 87, 34603469.

59. Dunbrack, R. L. (2002) Rotamer libraries in the 21st century, Curr. Opin. Struct. Biol. 12, 431-440.

60. McIntosh, T. J., and Simon, S. A. (2006) Roles of bilayer material properties in function and distribution of membrane proteins, Аnпи. Rev. Biophys. Biomol. Struct. 35, 177-198.

61. Demmers, J. A. A., van Duijn, E., Haverkamp, J., Greathouse, D V., Koeppe, R. E., II, Heck, A. J. R., and Killian, J. A. (2001) Interfacial positioning and stability of transmembrane peptides in lipid bilayers studied by combining hydrogen/deuterium exchange and mass spectrometry, J. Biol. Chem. 276, 34501-34508.

62. Lookene, A., Groot, N. B., Kastelein, J. J. P., Olivecrona, G., and Bruin, T. (1997) Mutation of tryptophan residues in lipoprotein lipase: Effects on stability, immunoreactivity, and catalytic properties, J. Biol. Chem. 272, 766-772.

63. Ridder, A. N. J. A., Morein, S., Stam, J. G., Kuhn, A., de Kruijff, B., and Killian, J. A. (2000) Analysis of the role of interfacial tryptophan residues in controlling the topology of membrane proteins, Biochemistry 39, 6521-6528.

64. Draheim, R. R., Bormans, A. F., Lai, R.-Z., and Manson, M. D. (2005) Tryptophan residues flanking the second transmembrane helix (TM2) set the signaling state of the Tar chemoreceptor, Biochemistry 44, 1268-1277.

65. Draheim, R. R., Bormans, A. F., Lai, R.-Z., and Manson, M. D. (2006) Tuning a bacterial chemoreceptor with protein-membrane interactions, Biochemistry 45, 14655-14664.

BI702235K 